Reprod. Nutr. Dévelop., 1985, 25 (1 B), 329-330.

\title{
Evolution du métabolisme hépatique des acides gras chez le rat au cours du sevrage
}

\author{
J. F. DECAUX, P. FERRÉ, P. ROBIN, D. ROBIN, J. R. GIRARD
}

Centre de Recherches sur la Nutrition, C.N.R.S.,

9, rue Jules-Hetzel, 92190 Meudon-Bellevue, France.

Chez le rat, comme chez la plupart des mammifères, le sevrage s'accompagne d'un changement brutal des conditions nutritionnelles. En effet, pendant la période d'allaitement, le rat reçoit de la mère un régime hyperlipidiquehypoglucidique : le lait. Au cours du sevrage, l'animal passe d'un régime hyperlipidique à une alimentation hyperglucidique-hypolipidique. Chez l'animal allaité, la consommation du régime lacté entraîne la nécessité d'effectuer une $\beta$-oxydation hépatique intense ainsi qu'une gluconéogenèse (formation de novo de glucose à partir de précurseurs comme le lactate et les acides aminés) élevée, alors que la lipogenèse est basse. Au moment du sevrage, le changement brutal de régime provoque des modifications du métabolisme hépatique dont la cinétique et les facteurs de régulation sont mal connus (Page et al., 1971 ; Yeh et Zee, 1976 ; Foster et Bailey, 1976 ; Benito et al., 1979). C'est pourquoi nous avons étudié l'évolution des capacités de $\beta$-oxydation, de lipogenèse et de gluconéogenèse à l'aide d'hépatocytes isolés chez des rats âgés de 15, 19 et 30 jours.

Le sevrage a été effectué à 19 jours en séparant la mère des petits et en leur offrant soit un régime croquettes de laboratoire (calories : glucides $65 \%$, lipides $11 \%$ ), soit un régime hyperlipidique (calories : glucides $18 \%$, lipides $55 \%$ ). Afin de tenir compte de l'augmentation d'un facteur 3 de la taille des hépatocytes entre 15 et 30 jours après la naissance, les résultats ont été exprimés par $\mathrm{mg}$ de protéines hépatiques totales (P.T.). Avec le régime hyperglucidique, la cétogenèse mesurée à l'aide d'oléate $0,40 \mathrm{mM}$ diminue d'un facteur $3[15 \mathrm{j}$ : $128 \pm 5,7(n=20)$ et $30 \mathrm{j}: 49,4 \pm 2,9(\mathrm{n}=16) \mathrm{nmol}$ oleate oxydé/h/mg P.T.]. Le rapport oléate oxydé/oléate estérifié et la gluconéogenèse chutent dès le 19 jour alors que la lipogenèse, mesurée à l'aide d'eau tritiée, indétectable à 15 jours, est élevée à 30 jours $[25,0 \pm 1,7 \mathrm{nmol}$ eau tritiée incorporée $/ \mathrm{h} / \mathrm{mg}$ P.T. $(n=7)$ ]. Les résultats obtenus suggéraient que, comme chez l'adulte, la régulation de l'oxydation des acides gras pouvait s'effectuer au niveau de la carnitine acyltransférase I par l'intermédiaire du malonylCoA (McGarry et al., 1977), métabolite intermédiaire de la voie de la lipogenèse. Ainsi, lorsque la lipogenèse est basse (cas de l'adulte à jeun), la concentration en malonylCoA est faible et les acyls CoA peuvent entrer dans la mitochondrie pour y être oxydés ; par contre, lorsque la lipogenèse est intense (cas de l'adulte nourri sur régime hyperglucidi- 
que), la concentration en malonylCoA est élevée et inhibe l'entrée des acides gras dans la mitochondrie (McGarry et al., 1978 ; McGarry et Foster, 1979). Chez les animaux de 30 jours nourris sur régime hyperglucidique, l'inhibition de la lipogenèse au niveau de la formation du malonylCoA par l'acide-5-(tetradecyloxy)-2furoïque (Benito et al., 1979) $[4,0 \pm 1,4 \mathrm{nmol}$ eau tritiée/h/mg P.T. ( $\mathrm{n}=7$ )] ne permet pas de restaurer des valeurs de $\beta$-oxydation comparables à celles obtenues 15 jours après la naissance. Ceci suggère qu'il existe au moment du sevrage des variations de la capacité intrinsèque des mitochondries à oxyder les acides gras.

Une étude sur mitochondries isolées de l'oxydation de dérivés des acides gras entrant à différents niveaux de la voie métabolique, indique que la diminution de la cétogenèse au moment du sevrage est effectivement liée à une diminution des capacités intrinsèques de la $\beta$-oxydation mitochondriale.

Le sevrage sur un régime hyperlipidique permet de maintenir jusqu'à 30 jours une cétogenèse et une gluconéogenèse élevées tandis que la lipogenèse reste basse. Ceci suggère que le régime hyperglucidique est un facteur déclenchant les variations métaboliques observées au cours du sevrage.

En conclusion, les modifications du métabolisme hépatique observées au cours du sevrage, liées au passage d'une alimentation lactée hyperlipidique hypoglucidique à une alimentation solide hyperglucidique hypolipidique, font intervenir des variations des capacités intrinsèques de $\beta$-oxydation de la mitochondrie. Les variations métaboliques observées sont liées à la consommation du régime hyperglucidique.

$10^{e}$ Réunion du groupe Développement I.N.R.A., Rennes, 9-10 mai 1984.

\section{Références}

BENITO M., WHITELAW E., WILLIAMSON D. H., 1979. Regulation of ketogenesis during the suckling-weanling transition in the rat. Biochem. J., 180, 137-144.

FOSTER P. C., BAILEY E., 1976. Changes in the activities of the enzymes of hepatic fatty acid oxidation during development of the rat. Biochem. J., 154, 49-56.

McGARRY J. D., FOSTER D. W., 1979. In support of the roles of malonylCoA and carnitine acyltransferase $I$ in the regulation of hepatic fatty acid oxidation and ketogenesis. $J$. biol. Chem., 254, 8163-8168.

McGARRY J. D., MANNAERTS G. P., FOSTER D. W., 1977. A possible role for malonylCoA in the regulation of hepatic fatty acid oxidation and ketogenesis. J. clin. Invest., 60, 265-270.

McGARRY J. D., TAKABAYASHI Y., FOSTER D. W., 1978. The role of malonyICoA in the coordination of fatty acid synthesis and oxidation in isolated rat hepatocytes. J. biol. Chem., 253, 8294-8300.

PAGE M. A., KREBS H. A., WILLIAMSON D. H., 1971. Activities of enzyme of ketone body utilization in brain and other tissues of suckling rats. Biochem. J., 121, 49-53.

YEH Y. Y., ZEE P., 1976. Insulin: a possible regulator of ketosis in newborn and suckling rats. Pediat. Res., 10, 192-197. 